TECHNICAL NOTE

\author{
S.S. Lollis \\ A.C. Mamourian \\ T.J. Vaccaro \\ A.-C. Duhaime
}

\section{Programmable CSF Shunt Valves: Radiographic Identification and Interpretation}

SUMMARY: The programmable CSF shunt valve has become an important tool in hydrocephalus treatment, particularly in the NPH population and in pediatric patients with complex hydrocephalus. The purpose of this study is to provide a single reference for the identification of programmable shunt valves and the interpretation of programmable shunt valve settings. Four major manufacturers of programmable shunts agreed to participate in this study. Each provided radiographic images and legends for their appropriate interpretation. Issues of MR imaging compatibility for each valve are also discussed.

ABBREVIATIONS: $\mathrm{H}=$ high; $\mathrm{L}=$ low; $\mathrm{M}=$ medium; $\mathrm{NPH}=$ normal pressure hydrocephalus; $\mathrm{P} / \mathrm{L}=$ performance levels
$\mathbf{H}$ ydrocephalus affects between $1 \%$ and $2 \%$ of the population. ${ }^{1}$ It accounts for 70,000 hospital admissions annually and the placement of between 18,000 and 33,000 CSF shunts in the United States each year. ${ }^{2}$ Because one-third of all shunts fail within 1 year of placement and the manifestations of shunt failure are protean, patients with shunts frequently undergo radiographic evaluation. ${ }^{3}$ These evaluations include not only cross-sectional imaging with CT and MR imaging but also plain radiographs of the shunt system.

Most CSF shunts consist of 3 components: a ventricular catheter, a valve, and a distal catheter. A shunt is a completely internalized system, as opposed to an external ventricular drain in which a ventricular catheter drains to a collection system at the bedside. The catheter components of a shunt are made from Silastic (Dow Corning, Midland, Michigan), a form of rubber tubing resistant to breakdown in the body. They are frequently impregnated with radiopaque material to aid in their radiographic visualization. The ventricular catheter sits within 1 of the ventricular spaces in the brain, most commonly the right lateral ventricle. The ventricular catheter is connected to a valve that regulates flow. To counter a siphoning effect associated with upright posture, many shunt systems also include an antisiphon device; this reduces overdrainage when the patient is standing.

Historically, shunt valves permitted a fixed amount of CSF drainage. This required the surgeon to select a specific valve for implantation, and if overdrainage or underdrainage resulted, a second operation was required to change the valve. The programmable valve is an important advancement in shunt technology. It provides the option of changing the opening pressure of the valve transcutaneously, most commonly with a device using a coded magnetic field. Although most valves placed in the United States are still fixed-pressure valves, the programmable valve has become an important tool in hydrocephalus treatment, particularly in the NPH population and in pediatric patients with complex hydrocephalus. ${ }^{4}$

Received June 18, 2009; accepted after revision July 21.

From the Sections of Neurosurgery (S.S.L., A.-C.D.) and Pediatric Radiology (T.J.V.), Dartmouth-Hitchcock Medical Center, Lebanon, New Hampshire; and Section of Neuroradiology (A.C.M.), Hospital of the University of Pennsylvania, Philadelphia, Pennsylvania.

Please address correspondence to S. Scott Lollis, MD, Section of Neurosurgery, DartmouthHitchcock Medical Center, One Medical Center Dr, Lebanon, NH 03756; e-mail: scott.lollis@ hitchcock.org

indicates article with supplemental on-line figures

DOl 10.3174/ajnr.A1997
Patients with NPH often require multiple adjustments of opening pressure to optimize cognitive function and gait stability, while avoiding overdrainage and the secondary subdural effusion. Other patients with hydrocephalus have a very narrow therapeutic window between symptomatic overdrainage and symptomatic underdrainage. Because programmable valves allow noninvasive fine tuning of the opening pressure in these difficult patient populations, their use is increasing. While the dependability and relatively low cost of fixed-pressure valves will likely ensure their continued use, the programmable valve is now an important established tool in the treatment of hydrocephalus. It is important to be aware of the impact of the programmable valve on patient management and, specifically, of the altered significance of ventricular size change in these patients.

The purpose of this study was to provide a single reference containing radiographic depictions of the major programmable shunt valves in current use as well as information for accurate reporting of shunt valve settings. This will assist radiologists and other physicians in the complete assessment of skull radiographs in patients with shunts. We have also consolidated the current recommendations of the manufacturers regarding MR imaging compatibility of these valves.

\section{Materials and Methods}

Major shunt manufacturers were contacted and asked to participate in this study. Four companies currently producing $\geq 1$ programmable shunt valve agreed to participate: Aesculap (Tuttlingen, Germany), Codman/Johnson \& Johnson (Raynham, Massachusetts), Medtronic (Minneapolis, Minnesota), and Sophysa (Orsay, France). The manufacturers provided photographs and plain radiographs of programmable shunt valves in current production as well as the appropriate legends describing the appearance of the valves at various settings. As of this date, these are the most commonly used programmable shunt valves in the United States. Each participating manufacturer provided written permission to publish the images herein.

\section{Results}

Figures 1-4 and On-line Figs 1-4 depict photographs and radiographs of shunt valves in common use in the United States as of 2009. All radiographs are depicted so that CSF flow is from the top of the image toward the bottom.

The Codman Hakim Programmable Valve (Codman/Johnson \& Johnson, Raynham, Massachusetts) permits regulation of the opening pressure between 30 and $200 \mathrm{~mm} \mathrm{H}_{2} \mathrm{O}$. Its function 

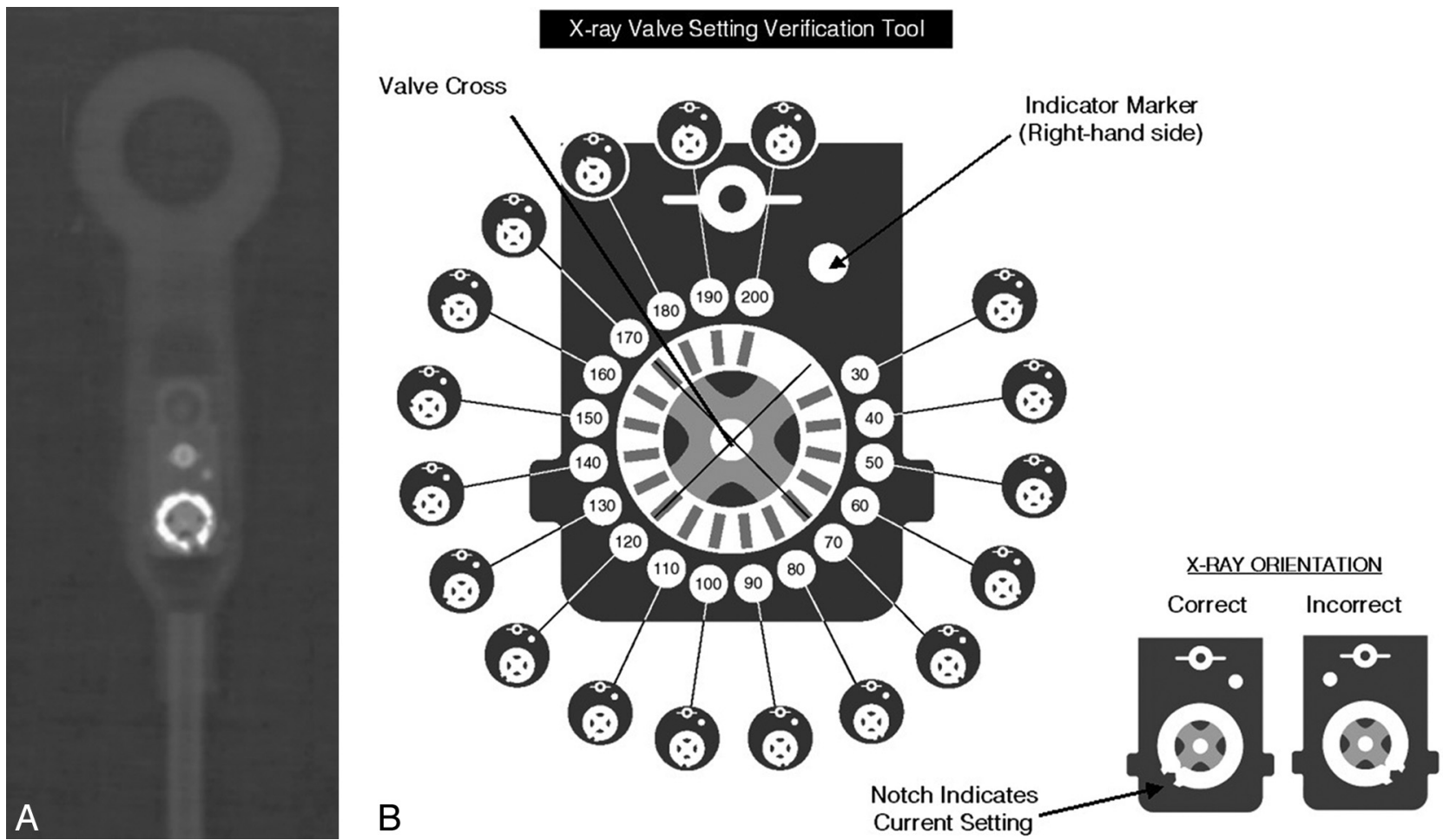

Fig 1. $A$, Radiographic appearance of the Codman Hakim Programmable Valve (set to $90 \mathrm{~mm} \mathrm{H}_{2} \mathrm{O}$ ). $B$, Setting code for the Codman Hakim Programmable Valve. Reproduced with permission from Codman/Johnson \& Johnson.

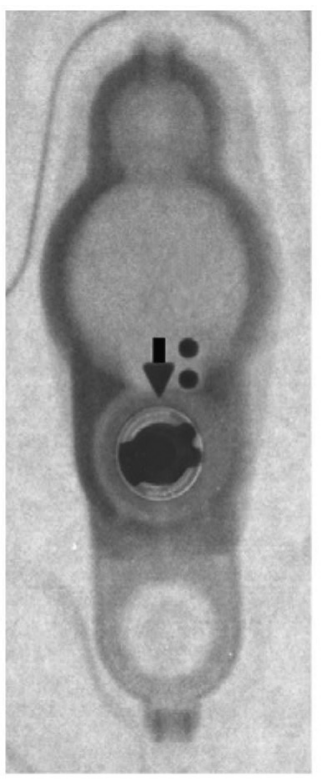

P/L 0.5

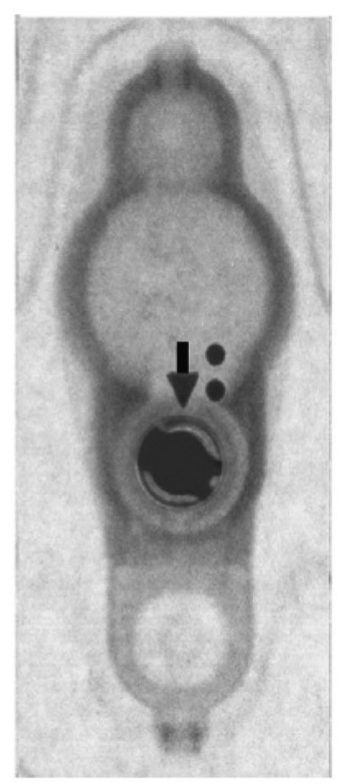

P/L 1.0

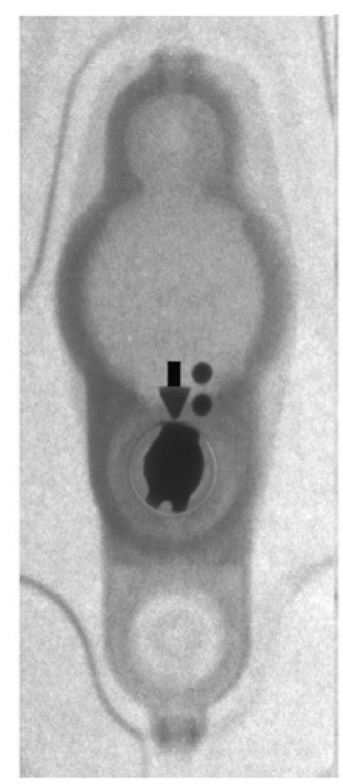

P/L 1.5

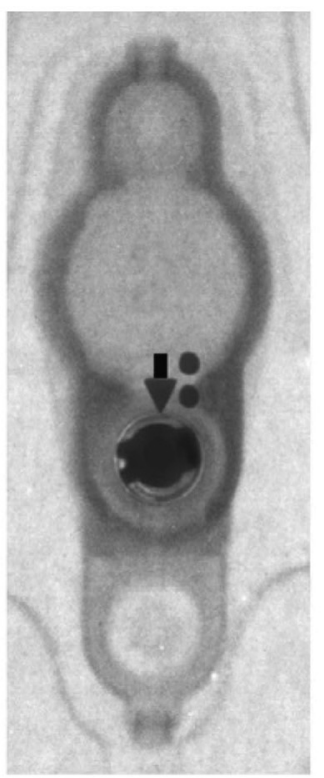

P/L 2.0

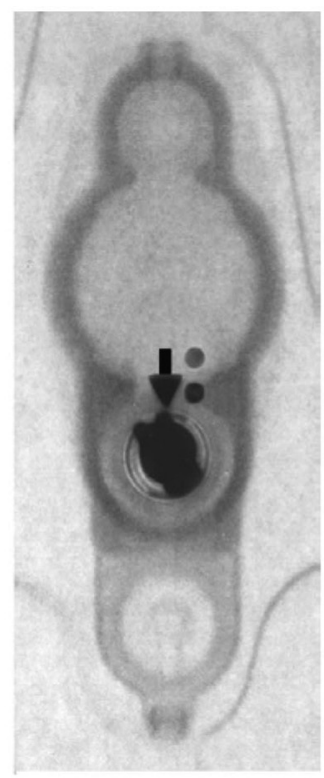

P/L 2.5

Fig 2. Medtronic Strata valve at various settings. Reproduced with permission from Medtronic Neurosurgery.

was reviewed in a recent article. ${ }^{5}$ The valve is often used in series with SiphonGuard (Codman/Johnson \& Johnson), an antisiphoning device intended to prevent overshunting during periods of upright posture (image available on-line at AJNR website). Figure $1 A$ depicts the radiographic appearance of a typical valve; the valve component consists of a hyperattenuated disk with a notched edge and 2 adjacent hyperattenuated circular structures. The valve setting is interpreted on the basis of the position of the notch relative to the 2 other circles (Fig $1 B$ ). Because the image is chiral, flipping the image to make it conform to the setting guide should not lead to misinterpretation. Current product literature states that patients with an implanted Codman Hakim Programmable Valve can safely undergo MR imaging under the following conditions: 1) static magnetic field of $\leq 3 \mathrm{~T}, 2$ ) spatial gradient of $\leq 720 \mathrm{G} / \mathrm{cm}$, and 3) limited radio-frequency energy to a wholebody-averaged specific absorption rate of 3 watts per kilogram for 


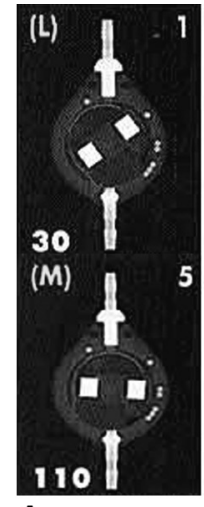

A
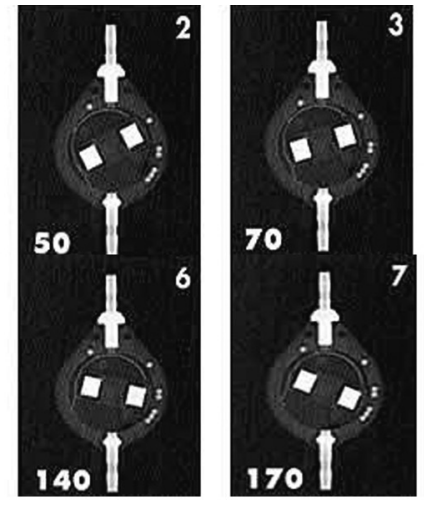

170

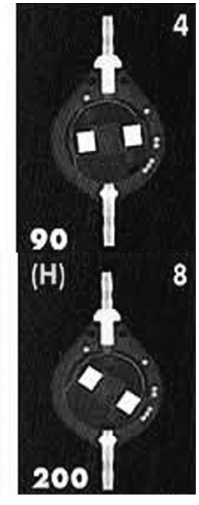

200
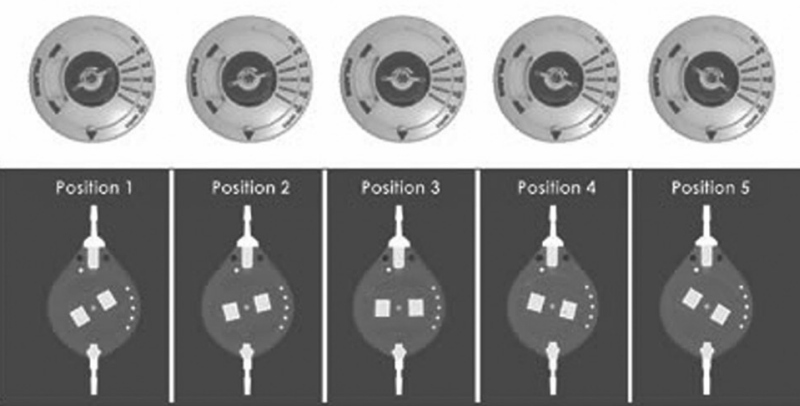

B

Fig 3. $A$, Setting code for the Sophysa Sophy SM8 valve. Operating pressure (millimeters of water) for each position is depicted in the lower left hand corner of each panel. $B$, Setting code for the Sophysa Polaris SPV valve. Operating pressures (millimeters of water) for positions 1 through 5 are 30, 70, 110, 150, and 200 mm $\mathrm{H}_{2} \mathrm{O}$, respectively. Reproduced with permission from Sophysa.

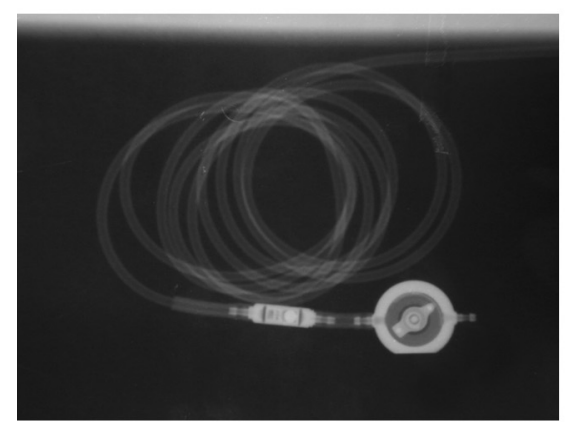

A
Aesculap Neurosurgery

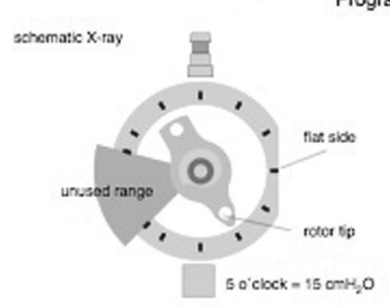

proGAV

Programmable valve with gravitational technology

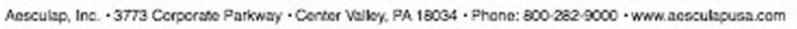

DCCegs $1 \mathrm{M} 307$

\begin{tabular}{|c|c|}
\hline $\begin{array}{c}\text { Opening pressure } \\
\text { for vertical posture }\end{array}$ & $\begin{array}{c}\text { Coding of } \\
\text { gravitational unit }\end{array}$ \\
\hline $10 \mathrm{cmH}_{2} \mathrm{O}$ & small, no ring \\
\hline $15 \mathrm{cmH}_{2} \mathrm{O}$ & large, no ring \\
\hline $20 \mathrm{cmH}_{2} \mathrm{O}$ & large, 1 ring \\
\hline $25 \mathrm{cmH}_{2} \mathrm{O}$ & large, 2 rings \\
\hline $30 \mathrm{cmH}_{2} \mathrm{O}$ & large, 3 rings \\
\hline $35 \mathrm{cmH}_{2} \mathrm{O}$ & large, 4 rings \\
\hline
\end{tabular}

Fig 4. $A$, Complete Aesculap Miethke proGAV assembly. B, Setting code for Aesculap Miethke proGAV adjustable unit. C, Setting code for Aesculap Miethke proGAV gravitational unit. Reproduced with permission from Aesculap, Inc.

a)

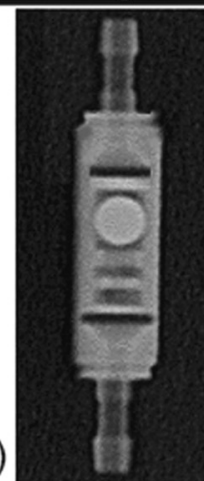

b)

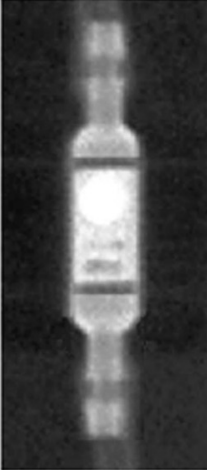

a) large, 2 rings $=25 \mathrm{cmH} 2 \mathrm{O}$,

b) $\mathrm{small}=10 \mathrm{cmH} 2 \mathrm{O}$

C 
15 minutes. Furthermore, it advises that the setting be checked on a plain film $\mathrm{x}$-ray after MR imaging to ensure that no change in opening pressure has occurred. ${ }^{6}$

The PS Medical Strata valve (Medtronic, Minneapolis, Minnesota) is an adjustable flow-control valve. The Strata valve has 5 settings or P/L, ranging from 0.5 to 2.5 (Fig 2). Each performance level corresponds to a range of opening pressures and flow rates; generally, a lower performance level corresponds to a lower opening pressure. The range of opening pressures is between 15 and $170 \mathrm{~mm} \mathrm{H}_{2} \mathrm{O}$. Multiple models of the Strata valve have been introduced, including the Strata II valve and the Strata small valve (images available on-line at AJNR website). All make use of the same radiographic scheme for setting assessment; the position of a notched disk relative to 2 small dots defines the P/L setting. Current product literature states that patients with Strata valves may undergo MR imaging by using a static field of $\leq 3 \mathrm{~T}$ but that inadvertent changes of the setting are possible. It advises that the setting be checked after MR imaging to ensure that this has not occurred. $^{7}$

Sophysa (Orsay, France) has developed 2 programmable valves, the Sophy and the Polaris. The Sophy valve permits a total of 8 positions; in the most common SM8 model, these positions represent opening pressures between 30 and $200 \mathrm{~mm} \mathrm{H}_{2} \mathrm{O}$. The valve setting is determined by the position of the rotating central rectangular structure relative to a fixed peripheral dot pattern (Fig $3 A$ ). Product literature states that the Sophy valve should have its setting checked after MR imaging to ensure that no inadvertent setting change has occurred. ${ }^{8}$ The Polaris valve is a newer valve, also developed by Sophysa. Figure $3 B$ depicts a Polaris valve at various settings; its peripheral dot pattern is slightly different from that of the Sophy valve. The Polaris valve permits a total of 5 positions; in the most common SPV model, these correspond to opening pressures between 30 and $200 \mathrm{~mm} \mathrm{H}_{2} \mathrm{O}$. Both the Sophy and Polaris valves come in different models, so the relationship between position and opening pressure is variable. Radiologists should, therefore, report the position (1-8 in the Sophy, 1-5 in the Polaris) and let the clinician familiar with the patient's system correlate this position with the appropriate opening pressure. Product literature states that the Polaris has been tested in MR imaging fields $\leq 3 \mathrm{~T}$ and that no inadvertent setting changes have been seen. ${ }^{9}$ The Polaris is also less susceptible to inadvertent setting changes by household magnets. A recent article tested multiple programmable valves for setting changes when placed in proximity to toy magnets; only the Polaris demonstrated consistent durability, with no setting alterations noted. ${ }^{7}$ Nevertheless, product literature advises that patients with either a Sophy or Polaris valve have their setting checked immediately after MR imaging.

The Miethke proGAV Programmable Shunt System (Aesculap, Tuttlingen, Germany) (Fig 4A) consists of 2 components in series, the adjustable unit and the gravitational unit. The adjustable unit is a circular structure with a rotating central pointer. The gravitational unit is a cylindrical structure. The adjustable unit can be changed to a pressure setting between 0 and $20 \mathrm{~cm}$ $\mathrm{H}_{2} \mathrm{O}$, as depicted in Fig $4 \mathrm{~B}$. The function of the gravitational unit is to prevent postural overdrainage. Its opening pressure gradually increases as the patient moves from a supine to an upright position. Multiple models of the gravitational unit are available, each with a different maximal opening pressure. This opening pressure of the gravitational component is determined by exam- ining the number of rings at the end of the cylinder ( $0-4$ rings) and the size of the component itself (small versus large); this setting code is depicted in Fig 4C. Complete reporting of a Miethke proGAV system requires the radiologist to report the setting of the adjustable unit and the opening pressure of the gravitational unit. In the vertical position, the true opening pressure is the sum of the 2 opening pressures; in the supine position, it is the opening pressure of the adjustable valve alone. A recent article assessed the safety of the proGAV system in relation to $3 \mathrm{~T} \mathrm{MR}$ imaging and found no excessive heating and no inadvertent setting changes ${ }^{10}$; however, in its current product literature, Aesculap recommends checking the setting of the valve after each MR imaging. ${ }^{11}$

\section{Discussion}

Patients who undergo ventriculoperitoneal shunt surgery with a programmable valve often undergo repeated adjustment of the shunt valve to optimize shunt function. Changes in ventricular caliber can be the result of shunt dysfunction or simply a change in the valve setting. For example, enlargement of the ventricles on serial cross-sectional imaging may be the result of obstruction in the shunt or an intentional increase in the setting of the shunt. Distinguishing between the 2 requires a working knowledge of valves in current use and an ability to interpret the valve setting on a plain radiograph. This brief communication provides radiologists and other physicians with a reference guide to programmable valves so that they may better assist clinicians in evaluating shunt function. It also provides important MR imaging safety information. All valves described herein are MR imagingcompatible up to 3T. However, they require prompt setting assessment and/or readjustment after MR imaging to correct changes in opening pressure induced by magnetic fields. Because an undetected change in opening pressure is potentially dangerous, it is prudent to confer with the clinician managing a given patient's shunt after each MR imaging.

\section{Acknowledgments}

We gratefully acknowledge the cooperation of Aesculap AG (Tuttlingen, Germany), Codman/Johnson \& Johnson (Raynham, Massachusetts), Medtronic (Minneapolis, Minnesota), and Sophysa (Orsay, France).

\section{References}

1. Greenberg MS. Handbook of Neurosurgery. 6th ed. New York: Thieme Medical Publishers; 2006

2. Bondurant CP, Jimenez DF. Epidemiology of cerebrospinal fluid shunting Pediatr Neurosurg 1995;23:254-58, discussion 259

3. Stein SC, Guo W. Have we made progress in preventing shunt failure? A critical analysis. J Neurosurg Pediatr 2008;1:40-47

4. Gallia GL, Rigamonti D, Williams MA. The diagnosis and treatment of idiopathic normal pressure hydrocephalus. Nat Clin Pract Neurol 2006;2:375-81

5. Schleipman AR, Bailey NO. Programmable shunt radiography. Radiol Technol 2001;73:108-16

6. Codman. Procedure Guide. Codman Hakim Programmable Valve system for hydrocephalus. http://www.codman.com/binary/org/CODMAN/PDF/VAL-10-001CHPV-proc-guide.pdf. Accessed May 22, 2009

7. Medtronic. MRI information. http://www.medtronic.com/neurosurgery/mri.html Accessed May 22, 2009

8. Sophy Adjustable Pressure Valve [package insert]. Orsay, France: Sophysa

9. Sophysa. Polaris adjustable valve: The MRI compatible valve. http://www. sophysa.com/en/?id=C3. Accessed May 22, 2009

10. Shellock FG, Habibi R, Knebel J. Programmable CSF shunt valve: in vitro assessment of MR imaging safety at 3T. AJNR Am J Neuroradiol 2006;27:661-65

11. proGAV instructions for use [package insert]. Tuttlingen, Germany: Aesculap; 2009 